\title{
Improved BSP Clustering Algorithm for Social Network Analysis
}

\author{
B. Saranya Preethi
}

\begin{abstract}
Social network analysis is a new research field in data mining. Social network analysis is the study of social networks to recognize the structure and behavior of friends. Social network analysis views social relationships in terms of network theory consisting of nodes and ties. The defining feature of social network analysis is its focus on the structure of relationships, ranging from casual acquaintance to close bonds. Social network analysis assumes that relationships are very essential. The main aspect of the social network analysis is clustering. The clustering in social network analysis is different from conventional clustering techniques. It needs grouping objects into classes depending on their links as well as their attributes. The conventional clustering approaches group objects only based on objects' similarity and it cannot be applied to social network analysis. So on the basis of BSP (Business System Planning) clustering algorithm, a social network clustering analysis algorithm is proposed. Moreover, before applying clustering, the Principal Component Analysis (PCA) technique is applied. Thus proposed algorithm, different from traditional BSP clustering algorithms, can group objects in a social network into different classes based on their links and identify relation among classes dynamically $\&$ require less amount of memory.
\end{abstract}

\section{Keywords--- Social Network, PCA, Clustering, Page Rank}

\section{INTRODUCTION}

A social network [1] is a social structure made up of individuals (or organizations) called "nodes", which are tied (connected) by one or more specific types of interdependency, such as friendship, kinship, common interest, financial exchange, dislike, sexual relationships, or relationships of beliefs, knowledge or prestige.

Social networks are highly dynamic, evolving relationships among people or other entities. This dynamic property of social networks makes studying these graphs a challenging task. A lot of research has been done recently to study different properties of these networks. Such complex analysis of large, heterogeneous, multi-relational social networks has led to an interesting field of study known as Social Network Analysis (SNA) [2].

The defining feature of social network analysis is its focus on the structure of relationships, ranging from casual acquaintance to close bonds [3,4]. Social network analysis assumes that relationships are important. It maps and measures formal and informal relationships to understand what

B. Saranya Preethi, Gobi Arts and Science College, Gobichettipalayam, E-mail:preethisharannmsc@gmail.com facilitates or impedes the knowledge flows that bind interacting units, viz., who knows whom, and who shares what information and knowledge with whom by what communication media (e.g., data and information, voice, or video communications). (Because these relationships are not usually readily discernible, social network analysis is somewhat akin to an "organizational x-ray".) Social network analysis is a method with increasing application in the social sciences and has been applied in areas as diverse as psychology, health, business organization, and electronic communications. More recently, interest has grown in analysis of leadership networks to sustain and strengthen their relationships within and across groups, organizations, and related systems.

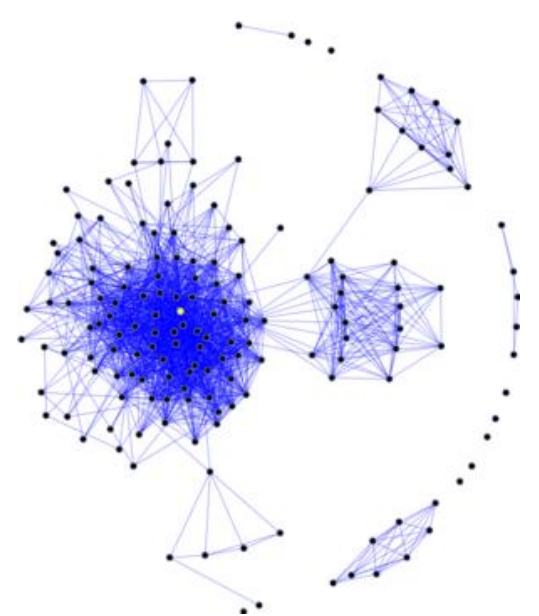

Figure 1: An Example of a Social Network Diagram. The Node with the Highest Betweenness Centrality is Marked in Yellow

Social network analysis, which can be applied to analysis of the structure and the property of personal relationship, web page links, and the spread of messages, is a research field in sociology. Recently social network analysis has attracted increasing attention in the data mining [5] research community. From the viewpoint of data mining, a social network is a heterogeneous and multi-relational dataset represented by graph [6].

This paper uses BSP clustering for social network analysis. The proposed approach uses Principal Component Analysis (PCA) which would provide better results. Principal component analysis is appropriate when you have obtained measures on a number of observed variables and wish to develop a smaller number of artificial variables (called principal components) that will account for most of the variance in the observed variables. The principal components may then be used as predictor or criterion variables in 
subsequent analyses.

\section{RELATED WORKS}

In this paper Prabhu et al., [7] propose an innovative clustering technique called the Rapid Clustering Method (RCM), which uses Subtractive Clustering combined with Fuzzy C-Means clustering along with a histogram sampling technique to provide quick and effective results for large sized datasets. Rapid Clustering Method can be used to cluster the dataset and analyze the characteristics in a social network. It can also be used to enhance the cross-selling practices using quantitative association rule mining.

In this paper, a social network analysis method based on ontology is proposed by Tao $\mathrm{Li}$ et al., [8], which can describe the semantic of relationship. We create the relationship ontology, and illuminate the attribute and constraints of ontology, so the computer can know the relationship semantic and implement the reasoning.

There is a great need to develop analytical methodology to analyze and to exploit the information contained in gene expression data. Because of the large number of genes and the complexity of biological networks, clustering is a useful exploratory technique for analysis of gene expression data. Other classical techniques, such as principal component analysis (PCA) [9], have also been applied to analyze gene expression data. Using different data analysis techniques and different clustering algorithms to analyze the same data set can lead to very different conclusions. The main goal is to study the effectiveness of principal components (PC's) in capturing cluster structure. In other words, we empirically compared the quality of clusters obtained from the original data set to the quality of clusters obtained from clustering the PC's using both real and synthetic gene expression data sets.

In this research, Sun Wen-jun and Qiu Hang-ming [11] employed the link analysis (LA) method on Sinapsilas VIP Blogosphere. After obtaining the link relationship of the Blogosphere members, we used the social network analysis (SNA) method to explore the structural features of the Blogosphere and the behavior patterns of its members. We also tried to map the link relationship shown in the Blogospheres to the real-life relationship and obtained good consistency.

Richi Nayak et al., [10] propose a social matching system that uses past relations and user similarities in finding potential matches. The proposed system is evaluated on the dataset collected from an online dating network. Empirical analysis shows that the recommendation success rate has increased to $31 \%$ as compared to the baseline success rate of $19 \%$.

\section{METHODOLOGY}

Principal Component Analysis (PCA) [12] in multivariate statistics is widely adopted as an effective unsupervised dimension reduction method and is extended in many different directions. The main justification of dimension reduction is that PCA uses singular value decomposition (SVD) which gives the best low rank approximation to original data in L2 norm due to Eckart-Young theorem. However, this essentially noise reduction perspective alone is inadequate to explain the effectiveness of PCA. In this paper, we provide a new perspective of PCA based on its close relationship with the Kmeans clustering algorithm. We show that the principal components are actually relaxed cluster membership indicators.

The central idea of Principal Component Analysis (PCA) is to reduce the dimensionality of the data set while retaining as much as possible the variation in the data set. Principal components (PC's) are linear transformations of the original set of variables. PC's are uncorrelated and ordered so that the first few PC's contain most of the variations in the original data se.

Principal component analysis (PCA) [15] is a classic tool for analyzing large scale multivariate data. It seeks linear combinations of the data variables (often called factors or principal components) that capture a maximum amount of variance. Numerically, PCA only amounts to computing a few leading eigenvectors of the data's covariance matrix, so it can be applied to very large scale data sets. One of the key shortcomings of PCA however is that these factors are linear combinations of all variables; that is, all factor coefficients (or loadings) are non-zero. This means that while PCA facilitates model interpretation and visualization by concentrating the information in a few key factors, the factors themselves are still constructed using all observed variables. In many applications of PCA, the coordinate axes have a direct physical interpretation; in finance or biology for example, each axis might correspond to a specific financial asset or gene. In such cases, having only a few nonzero coefficients in the principal components would greatly improve the relevance and interpretability of the factors. In sparse PCA, we seek a trade-off between the two goals of expressive power (explaining most of the variance or information in the data) and interpretability (making sure that the factors involve only a few coordinate axes or variables). When PCA is used as a clustering tool, sparse factors will allow us to identify the clusters with the action of only afew variables

Some background on PCA. The original $\mathrm{n}$ data points in m-dimensional space is contained in the data matrix $\left(x_{1}, \ldots, x_{n}\right)=X$. In general data is not centered around the origin. We define the centered data matrix

$$
Y=\left(y_{1}, \ldots, y_{n}\right) \text {, where } y_{i}=x_{i}-\bar{x} \text { and } \bar{x}=\sum_{i} \frac{x_{i}}{n} \text {. }
$$

The covarance matrix is given by

$$
S=\sum_{i}\left(x_{i}-\bar{x}\right)\left(x_{i}-\bar{x}\right)^{T}=Y Y^{T}
$$

The principal eigenvectors $u_{k} \circ Y Y^{T}$ are the principal directions of the data Y. The principal eigenvectors $v_{k}$ of the Gram matrix $Y Y^{T}$ are the principal components; entries of each $v_{k}$ are the projected values of data points on the principal direction $u_{k} \cdot v_{k}$ and $u_{k}$ are related via: $v_{k}=Y^{T} \frac{u_{k}}{\lambda_{k}^{1 / 2}}:$ where $\lambda_{k}$ is the eigenvalue of the covarance matrix $Y Y^{T}$. 
Thus after applying PCA to the data, the principal components are identified from the data to be clustered. Thus only the important and the vital data are going to be clustered. In this proposed approach, the social network is going to be analyzed based on the Business System Planning (BSP).

\section{Social Network Analysis Based on BSP Clustering}

Clustering analysis of social network is studied. In the second section, a social network clustering algorithm is proposed based on BSP clustering algorithm. The algorithm can group objects in a social network into different classes based on their links, and it can also identify the relations among classes. In the third section, an example of social network clustering algorithm is presented.

There has been extensive research work on clustering in data mining. Traditional clustering algorithms [3] divide objects into classes based on their similarity. Objects in a class are similar to each other and are very dissimilar from objects in different classes. Social network clustering analysis, which is different from traditional clustering problem, divides objects into classes based on their links as well as their attributes. The biggest challenge of social network clustering analysis is how to divide objects into classes based on objects' links, thus find algorithms that can meet this challenge.

The BSP (business system planning) clustering algorithm [13] is proposed by IBM. It designed to define information architecture for the firm in business system planning. This algorithm analyses business process and their data classes, cluster business process into subsystems, and define the relationship of these sub-systems.

Basically BSP clustering algorithm uses objects (business processes) and links among objects (data classes) to make clustering analysis. Similarly social network also includes objects and links among these objects. In view of the same pre-condition, the BSP clustering algorithm can be used in social network clustering analysis [14]. According to graph theory, social network is a directed graph composed by objects and their relationship. Figure 1 shows a sample of social network, the circle in the figure represents an object; the line with arrow is an edge of the graph, and it represents directed link between two objects, so a social network is a directed graph.

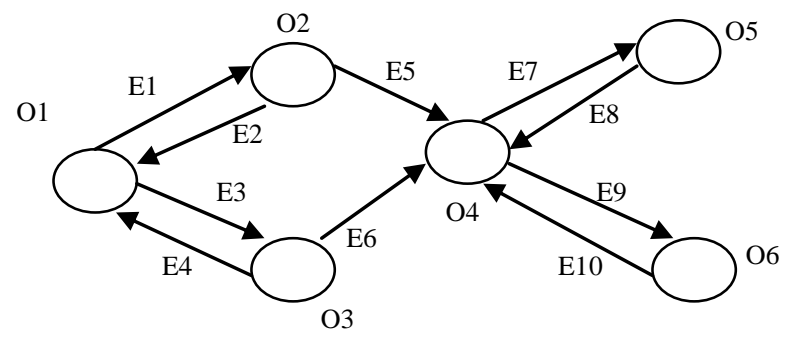

Figure 2: A Sample of Social Network

In figure 2, Let $O i$ be an object in social network $(i=1 \ldots . m$ ), let $E j$ which means directed link between two objects, be a directed edge of the graph $(j=1 \ldots n)$.
After definition of objects and directed edges, also define reachable relation between two objects. There are two kinds of reachable relation among objects, shown as following:

1) One-step reachable relation: if there has directed link from $O i$ to $O j$ through one and only one directed edge, then $\mathrm{O} i$ to $O j$ is a one-step reachable relation. For instance in figure 1 there has a directed link from $O 1$ to $O 2$ through the directed edge $E 1, O 1$ to $O 2$ is one-step reachable relation.

2) Multi-steps reachable relation: if there has directed link from $O i$ to $O j$ through two or more directed edges, then $O \mathrm{i}$ to $O j$ is a multi-steps reachable relation. For instance in figure 1 has a directed link from $O 1$ to $O 4$ through directed edges $E 1$ and $E 5$, then $\mathrm{O} 1$ to $\mathrm{O} 4$ is a 2 -steps reachable relation.

After these definitions, use BSP clustering algorithm to analyses a social network. The analysis processes are as following steps:

\section{Generate Edge Creation Matrix and Edge Pointed Matrix}

First according to the objects and edges in the graph, define two matrixes $L c$ and $L p$. Let $L c$ be a $m \times n$ matrix which means the creation of edges. In the matrix, $L c(i, j)=1$ denotes object $O i$ connects with the tail of edge $E j$, which means that object $O i$ creates the directed edge $E j . L(i, j) c=0$ denotes $O i$ doesn't connect with the tail of edge $E j$, which means $E j$ isn't created by object $O i$.

Let $L p$ be a $m \times n$ matrix which means the pointed relations of edges. In the matrix, $L(i, j) p=1$ denotes object $O i$ connects with the head of edge $E j$, which means object $O i$ is pointed to by the directed edge $E j . L(i, j) p=0$ denotes $O i$ doesn't connect with the head of edge $E j$, which means $E j$ doesn't point to $\mathrm{Oi}$.

\section{Calculate One-Step Reachable Matrix between Objects}

After the definition of $L c$ and $L p$, calculate one-step reachable matrix between objects through the following equation.

$$
\begin{gathered}
G=L_{c} * L_{p}^{T}=g_{i, j}={ }_{k=1}^{n} V\left(1_{c}(i, k)^{\wedge} 1_{p}^{T}(k, j)\right. \\
i=1 \ldots \ldots m,[j=1 \ldots \ldots m]
\end{gathered}
$$

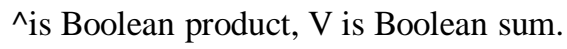

$G(i, j)=1$ means $O i$ to $O j$ is a one-step reachable relation, $G(i, j)=0$ means there hasn't a one-step reachable relation from $O i$ to $O j$. Through $\mathrm{G}$, calculate all one-step reachable relation between objects.

\section{Calculate Multi-Steps Reachable Matrix Between Objects}

Besides one-step reachable relation, there are multi-steps reachable relations between objects too. Also need calculate multi-steps reachable matrices (2-steps, 3-steps, ...,m-1-steps).

According to graph theory and the BSP clustering algorithm, calculate multi-steps reachable matrix G2, G3, $\mathrm{G} 4, \ldots, G m^{-1}$. Following equations show the calculation of multi-steps reachable matrix: 


$$
\begin{gathered}
G^{2}=G * G=g_{i, j}^{2}={ }_{k=1}^{m} V\left(g(i, k)^{\wedge} g(k, j)\right) \\
i=1 \ldots \ldots m,[j=1 \ldots \ldots m] \\
G^{3}=G^{2} * G \\
G^{4}=G^{3} * G \\
G^{m-1}=G^{m-2} * G
\end{gathered}
$$

These matrices include 2-steps, 3-steps... m-1-steps reachable relations between objects.

Now $n$-steps reachable relation between two objects through $G 2, G 3, G 4, \ldots, G m-1$.

\section{Calculate Reachable Matrix}

Because only consider whether reachable relations exist between two objects, but do not care these relations are onestep or multi-steps, so calculate reachable matrix $R$ based on $G, G 2, G 3, G 4, \ldots, G m-1$. The calculation of $R$ is shown as following equation:

\section{$R=I \mathrm{~V} G \mathrm{VG} 2 \ldots \mathrm{VGm}-1$}

$\mathrm{V}$ is Boolean sum, $I$ is unit matrix.

$R(i, j)=1$ means reachable relation exists from $O i$ to $O j$, but the reachable relations existing in matrix $R$ is not mutual, for instance $R(i, j)=1$ means reachable relation exists from $O i$ to $O j$, but it doesn't means reachable relation exists from $O j$ to $O i$. Mutual reachable relations between two objects are important in a social network, so calculate mutual reachable matrix based on $R$.

\section{Calculate Mutual Reachable Matrix and Generate Clusters}

The mutual reachable matrix can be calculated through following calculate equation.

\section{$Q=R^{\wedge} R T$}

${ }^{\wedge}$ means Boolean product

In the matrix $Q(i, j)=1$ means there are mutual reachable relation between $O i$ and $O j$. In a social network if two objects that have mutual reachable relation, they should belong to the same class, thus cluster based on $Q$.

Thus according to mutual reachable matrix $Q$, divide a social network into classes based on strong submatrices in $Q$ or adjusted $Q$. While strong sub-matrix is defined as follows.

Strong sub-matrix: if all elements in a sub-matrix of $Q$ are 1 , this sub matrix is strong submatrix.

\section{Identify Relationships among Classes}

After clustering of social network, also need identify relationship among clusters. This can be done through generated clusters and one-step reachable matrix $G$. If there is one-step reachable relation between two objects in different classes, so directed links exist between classes. Through $G$, identify all relations among classes.

After pervious 6 steps, divide a social network into classes. Social network clustering analysis algorithm can be given:\
$Q^{-}>C k$ means generating clusters through mutual reachable matrix $Q$, and $(C k, Q$ )- $>\operatorname{Relation}(C k)$ means identifying relationships among clusters base on clusters and one-step reachable matrix $G$.

\begin{tabular}{l}
\hline Input \\
$L_{c}:$ Edge creation Matrix \\
$L_{p}:$ Edge pointed matrix \\
Begin \\
$G=L_{c} * L_{u}^{T}$ \\
For $\mathrm{k}=3$ to $\mathrm{m}$ do \\
$\mathrm{Gk}-1=\mathrm{Gk}-2 * \mathrm{G}$ \\
$\mathrm{R}=\mathrm{IVGVG} 2 \ldots . . \mathrm{Gm}-1$ \\
$\mathrm{Q}=\mathrm{R}^{\wedge} \mathrm{R}^{\mathrm{T}}$ \\
$\mathrm{Q}_{\mathrm{k}}->\mathrm{Relation}\left(\mathrm{C}_{\mathrm{k}}\right)$ \\
End
\end{tabular}

\section{Improvement over BSP Clustering Algorithm}

In previous paper based on BSP clustering algorithm, an algorithm of social network clustering analysis is proposed. It divides a social network into different classes according to objects in the social network and links between objects, and it also can identify relations among clusters.

Main disadvantage of this algorithm is that it uses matrices to store edges and reachable relations, in a real social network these matrices will be very huge, can't load into main memory. But because these matrices are very sparse, so design an efficient data structure to overcome this shortcoming.

Present paper propose modification of existing BSP algorithm using Link list data structure. Using this data structure can overcome shortcoming (which have been mention above). Following procedure is require for converting this sparse metrix in to link list:

A matrix is a two-dimensional data object made of $m$ rows and $n$ columns, therefore having $m, n$ values. When $m=n$, call it a square matrix.

The most natural representation is to use two-dimensional array $\mathrm{A}[\mathrm{m}][\mathrm{n}]$ and access the element of ith row and jth column as $A[i][j]$. If a large number of elements of the matrix are zero elements, then it is called a sparse matrix.

Representing a sparse matrix by using a two-dimensional array leads to the wastage of a substantial amount of space. Therefore, an alternative representation must be used for sparse matrices.

One such representation is to store only non-zero elements along with their row positions and column positions. That means representing every non-zero element by using triples (i, $\mathrm{j}$, value), where $\mathrm{i}$ is a row position and $\mathrm{j}$ is a column position, and store these triples in a linear list. It is possible to arrange these triples in the increasing order of row indices, and for the same row index in the increasing order of column indices. Each triple (i,j,value) can be represented by using a node having four fields as shown in the following: 
Struct snode \{

Int row,col,val;

Struct snode *next;

\} ;

\begin{tabular}{|l|l|l|l|}
\hline Row & Col & Val & $*_{\text {next }}$ \\
$\longrightarrow$
\end{tabular}

1. In order to add two sparse matrices represented using the sorted linked lists as shown in the preceding program, the lists are traversed until the end of one of the lists is reached.

2. In the process of traversal, the row indices stored in the nodes of these lists are compared. If they don't match, a new node is created and inserted into the resultant list by copying the contents of a node with a lower value of row index. The pointer in the list containing a node with a lower value of row index is advanced to make it point to the next node.

3. If the row indices match, column indices for the corresponding row positions are compared. If they don't match, a new node is created and inserted into the resultant list by copying the contents of a node with a lower value of column index. The pointer in the list containing a node with a lower value of column index is advanced to make it point to the next node.

4. If the column indices match, a new node is created and inserted into the resultant list by copying the row and column indices from any of the nodes and the value equal to the sum of the values in the two nodes.

5. After this, the pointers in both the lists are advanced to make them point to the next nodes in the respective lists. This process is repeated in each iteration. After reaching the end of any one of the lists, the iterations come to an end and the remaining nodes in the list whose end has not been reached are copied, as it is in the resultant list.

After pervious 5 steps, divide a social network into classes. Social network clustering analysis algorithm can be given:

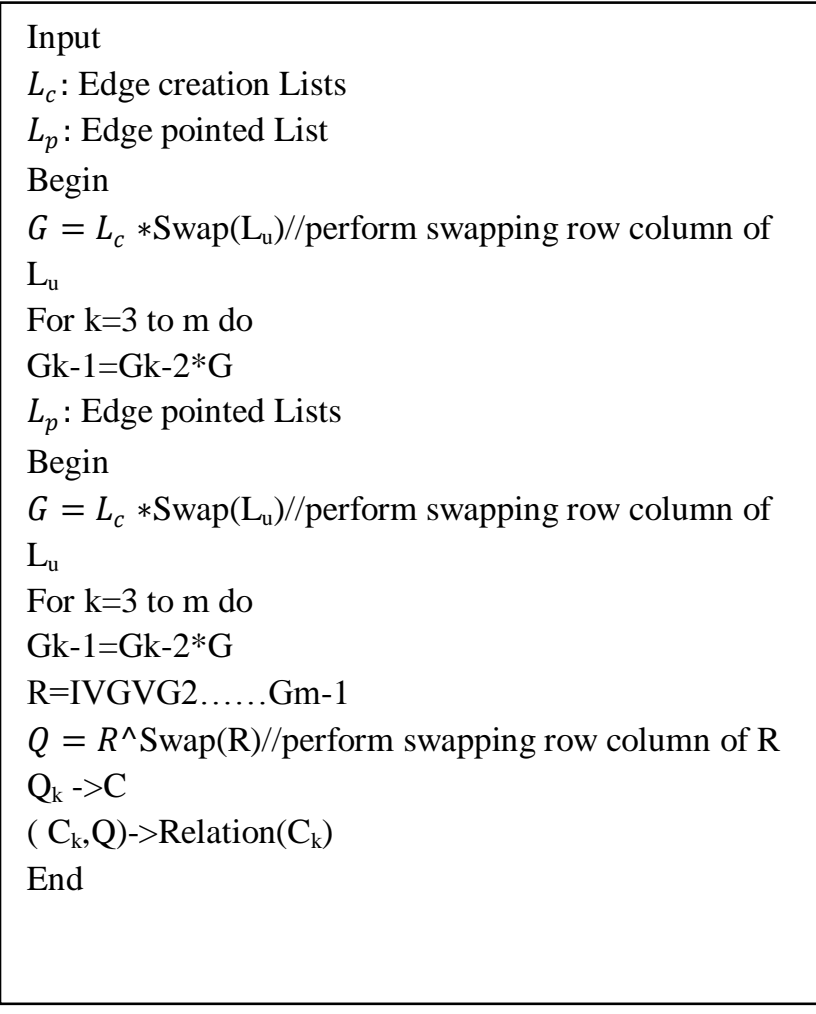

\section{EXPERIMENTAL RESULTS}

The performance of the proposed social network using the PCA is evaluated on the Zachary's Karate Club dataset. It is the data set which is a social network of friendships between 34 members of a karate club at a US university in the 1970.

The proposed PCA and BSP clustering is compared with the existing BSP clustering algorithm. The performance of the proposed system is evaluated based on the parameters like clustering accuracy and classification time.

\section{Classification Accuracy}

The proposed web personalization uses the modified fuzzy possibilistic c means.

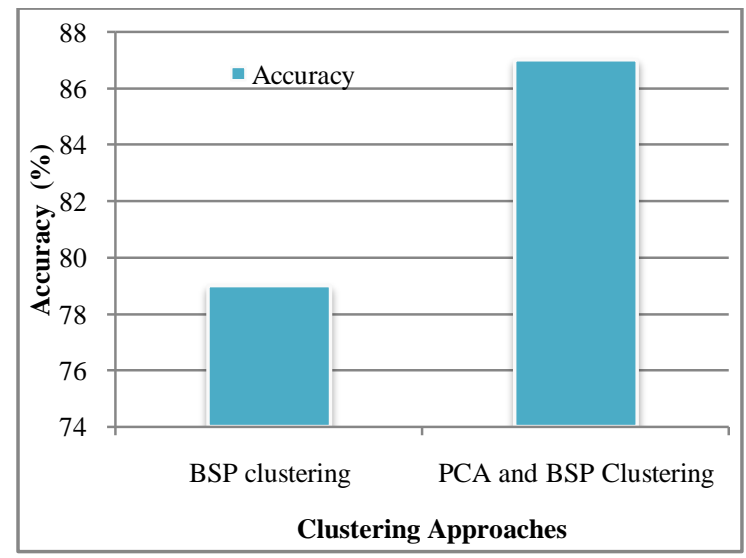

Figure 3: Comparison of the Classification Accuracy

The accuracy of the proposed web personalization system 
is compared with the previous web personalization systems which uses the fuzzy c means for clustering. It is observed from the figure 3 that the classification accuracy of the proposed approach using PCA and BSP approach is very high when compared to the BSP approach.

Time Taken For Classification

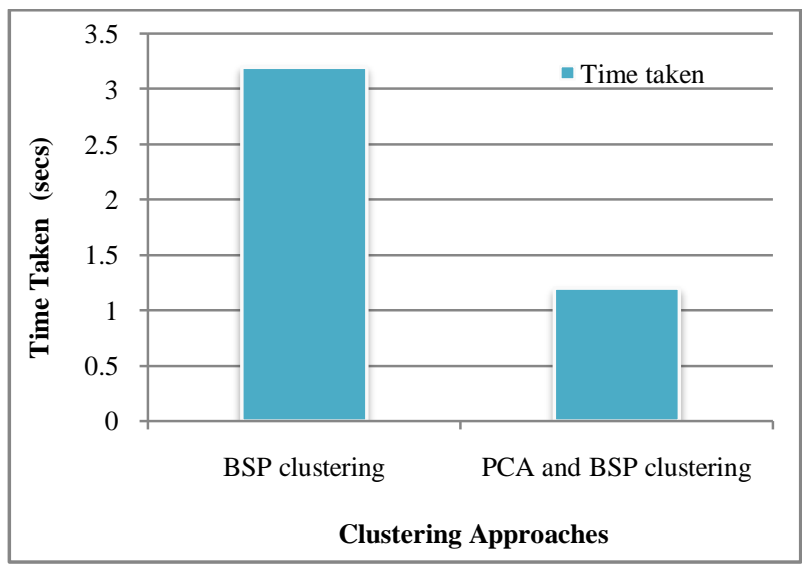

Figure 4: Comparison of the Classification Time

Figure 4 shows the graphical representation of the comparison of the time taken for the clustering classification of the clustering approaches.

It is observed from the graph that the time taken by proposed approach using PCA and BSP approach is very less when compared to the existing BSP approach.

\section{CONCLUSION}

This paper focused on the analysis of social network using Principal Component Analysis (PCA) with BSP clustering algorithm. This proposed approach divides a social network into different classes according to objects in the social network and links between objects, and it also can identify relations among clusters. The main contribution of this research work is the integration of Principal Component Analysis (PCA) technique with the BSP algorithm. The performance of the proposed approach is evaluated on the Zachary's Karate Club dataset. The performance of the proposed approach is compared with the existing BSP clustering approach. Classification accuracy and classification time are the two parameters considered for the evaluation of the proposed approaches. From the experimental observation, it is clearly observed that the proposed BSP clustering with PCA shows significant performance in terms of accuracy and classification time. Thus the proposed approach is very significant in providing better social network analysis.

\section{REFERENCES}

[1] Jamali, M. and Abolhassani, H.; "Different Aspects of Social Network Analysis", pages 66-72, 2007.

[2] J. Srivastava.; "Data mining for social network analysis", IEEE International Conference on Intelligence and Security Informatics, 2008.

[3] Wei Xue, JuWei Shi and Bo Yang, "X-RIME: Cloud-Based Large Scale Social Network Analysis", Pages: 506 - 513, 2010.

[4] Aiwu Xu and Xiaolin Zheng, "Dynamic Social Network Analysis Using Latent Space Model and an Integrated Clustering Algorithm", Eighth
IEEE International Conference on Dependable, Autonomic and Secure Computing, Page(s): 620 - 625, 2009.

[5] Han J, Kamber M. "Data Mining: Concepts and Techniques 2nd edition San Francisco: The Morgan Kaufmann Publishers, 2006.

[6] Kubica J, Moore A and Schneider J. "Tractable Group Detection on Large Link Data Sets", Proceeding 3rd IEEE international conference on data mining, Melbourne, FL, pages 573-576, 2003.

[7] Prabhu, J.; Sudharshan, M.; Saravanan, M.; Prasad, G.; "Augmenting Rapid Clustering Method for Social Network Analysis", 2010 International Conference on Advances in Social Networks Analysis and Mining (ASONAM), Page(s): 407 - 408, 2010.

[8] Tao Li; He Yang; Jun He; Yong Ai; "A Social Network Analysis methods based on ontology", 2010 3rd International Symposium on Knowledge Acquisition and Modeling (KAM), Page(s): 258 - 261, 2010.

[9] Kose, K.; Cetin, A.E.; "Motion based clustering of model animations using PCA", 17th IEEE Signal Processing and Communications Applications Conference, 2009. SIU 2009. Page(s): 317 - 320, 2009.

[10] Richi Nayak, Meng Zhang and Lin Chen, "A Social Matching System for an Online Dating Network: A Preliminary Study", IEEE International Conference on Data Mining Workshops, 2010.

[11] Sun Wen-jun and Qiu Hang-ming; "A social network analysis on Blogospheres", Proceedings., International Conference on Management Science and Engineering, 2008. ICMSE 2008. 15th Annual Conference, Page(s): 1769 - 1773, 2008.

[12] Yeung, K. Y. and Ruzzo, W. L., "An empirical study of Principal Component Analysis for clustering gene expression data", 2001.

[13] Communications of the IIMA (2007) Volume 7 Issue 4 "Social Network Analysis Based on BSP Clustering Algorithm" Gong Yu School of Business Administration China University of Petroleum.

[14] Yeung, K. Y. and Ruzzo, W. L., "An empirical study of Principal Component Analysis for clustering gene expression data", 2001.

[15] Jiangtao Qiu, Zhangxi Lin, Changjie Tang and Shaojie Qiao, "Discovering Organizational Structure in Dynamic Social Network", Ninth IEEE International Conference on Data Mining, 2009. 